\title{
Variabilidade espacial de atributos físicos de um Latossolo Vermelho sob cultivo de cana-de-açúcar ${ }^{1}$
}

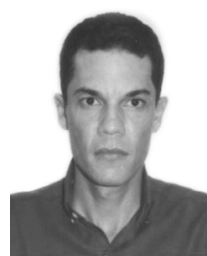

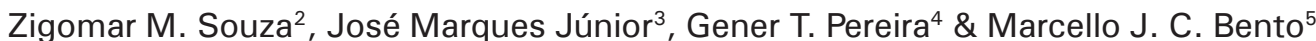

${ }^{1}$ Parte da tese de Doutorado do primeiro autor, financiada pela Fapesp

2 FCAV/UNESP. Prof. Paulo Donato Castellane s/n, CEP 14870-900, Jaboticabal, SP. E-mail: zigomar@fcav.unesp.br (Foto)

${ }^{3}$ Departamento de Solos e Adubos, FCAV/UNESP

4 Departamento de Ciências Exatas, FCAV/UNESP

${ }^{5}$ Usina São Martinho, CEP 14850-000 Pradópolis, SP

Protocolo 192 - 17/12/2002 - Aprovado em 6/2/2004

\begin{abstract}
Resumo: 0 uso intensivo dos Latossolos no nordeste do Estado de São Paulo com a cana-deaçúcar, com preparo superficial excessivo e queima dos resíduos, modifica significativamente as propriedades físicas do solo. Este trabalho teve como objetivo avaliar a variabilidade espacial de atributos físicos de um Latossolo Vermelho eutroférrico sob cultivo de cana-de-açúcar, através da geoestatística. Os solos foram amostrados nos pontos de cruzamento de uma malha, com intervalos regulares de $10 \mathrm{~m}$, nas profundidades de $0-0,2 ; 0,2-0,4$ e 0,4-0,6 m, totalizando 100 pontos; em cada amostra foram determinadas porosidade total, macroporosidade, microporosidade e densidade do solo. $O$ coeficiente de variação indicou variabilidade baixa para porosidade total nas profundidades 0,2 - 0,4 e 0,4-0,6 m, microporosidade na profundidade de 0,2 - 0,4 $\mathrm{m}$ e densidade do solo em todas as profundidades estudadas, e média para microporosidade nas profundidades de $0-0,2$ e 0,4 - 0,6 m e porosidade total na profundidade de $0-0,2 \mathrm{~m}$, enquanto a macroporosidade apresentou variação alta. Os maiores alcances foram observados nas profundidades de 0,2 - 0,4 m e 0,4 - 0,6 $\mathrm{m}$ para todos os atributos estudados. Os altos valores para a densidade do solo, sobretudo na profundidade de 0,2 - 0,4 m, promoveram redução da porosidade total, microporosidade em particular, na macroporosidade, indicando uma compactação nesta camada. Pequenas variações nas formas do relevo condicionam variabilidade diferenciada para os atribultos físicos do solo.
\end{abstract}

Palavras-chave: geoestatística, paisagem, porosidade do solo, densidade do solo

\section{Spatial variability of physical attributes of Oxisol under sugarcane crop}

\begin{abstract}
The intensive use of the Oxisols in the northeast of São Paulo State with the sugarcane using extreme superficial preparation and burning of the residues significantly modifies the physical properties of the soil. This work had the objective to evaluate the spatial variability of physical attributes of an Oxisol under sugarcane crop, by applying the technique of geostatistics. The soils were collected at the grid points, with regular intervals of $10 \mathrm{~m}$, at the depths of $0-0.2,0.2-0.4$ and $0.4-0.6 \mathrm{~m}$, totaling 100 points. In each soil sample the total porosity, macroporosity, microporosity and bulk density of the soil were determined. The variation coefficient indicated low variability for the variables of total porosity in the layers $0.2-0.4$ and $0.4-0.6 \mathrm{~m}$, microporosity in the $0.2-0.4 \mathrm{~m}$ layer and bulk density in all layers and average variation for microporosity in the layers of $0-0.2$ and $0.4-0.6 \mathrm{~m}$ and total porosity in the $0-0.2 \mathrm{~m}$, while the macroporosity presented a high variation. The higher ranges were observed in the $0.2-0.4$ and $0.4-0.6 \mathrm{~m}$ layers for all the studied attributes. The high values for the variable bulk density, mainly in the layer of $0.2-0.4 \mathrm{~m}$, had promoted a reduction of the total porosity, mainly microporosity and in the macroporosity, indicating compaction of this layer. Small variations in the forms of the relief condition differentiated spatial variability for physical attributes.
\end{abstract}

Key words: geostatistics, landscape, porosity of the soil, bulk density

\section{INTRODUÇÃO}

A agricultura moderna tem, como fundamento, o desenvolvimento sustentável, que objetiva uma produtividade econômica sem degradar o ambiente. Uma exploração agrícola sustentável requer, entre outros fatores, a manutenção e/ou melhoria da qualidade do solo; porém, a intensificação da exploração agrícola, aliada ao uso e ao manejo inadequado do solo, tem provocado modificações negativas nas propriedades físicas do solo (Stone \& Silveira, 2001) aumentando sua erosão e reduzindo a produtividade das culturas (Schaefer et al., 2001). O uso intensivo dos Latossolos Vermelho eutroférrico no nordeste do Estado de São Paulo com a cana-de-açúcar, com preparo superficial excessivo e queima dos resíduos, modifica 
significativamente as propriedades físicas do solo. As principais alterações são evidenciadas pela diminuição do volume de macroporos, do tamanho de agregados, da taxa de infiltração de água no solo e pelo aumento da resistência à penetração de raízes e da densidade do solo (Albuquerque et al., 1995; Klein \& Boller, 1995; Cavenage et al., 1999; Utset \& Cid, 2001).

As propriedades físicas do solo desempenham importante papel, senão o principal dentre as propriedades do solo (Warrick $\&$ Nielsen, 1980). Neste sentido, a variabilidade espacial de suas propriedades deve ser bem conhecida, visando minimizar os erros na amostragem e no manejo do solo. O solo apresenta heterogeneidade, tanto vertical como horizontal, imposta pela natureza dos fatores responsáveis pela sua formação. Vários estudos relatam que a variabilidade das propriedades físicas do solo apresenta correlação ou dependência espacial (Reichardt, 1985; Machado, 1994; Carvalho et al., 2002; Carvalho et al., 2003). Portanto, a análise da variabilidade do solo por meio de técnicas da geoestatística, pode indicar alternativas de manejo, para reduzir os efeitos da variabilidade do solo sobre a produção das culturas (Trangmar et al., 1985).

Os atributos físicos do solo variam entre pontos relativamente próximos em área de mesma unidade taxonômica, muitas vezes de forma significativa. Em conseqüência dessas variações, o uso do valor médio é, às vezes, de pouca utilidade, podendo conduzir a decisões de manejo equivocadas, principalmente no sistema solo-água-planta (Macedo et al., 1998). O conhecimento das formas do relevo, identificadas em diferentes escalas temporais e espaciais, é de extrema importância. Li et al. (2001) e Vaidya \& Pal (2002), afirmam que a geoestatística pode auxiliar na avaliação da dependência espacial de atributos do solo na paisagem. Bathke \& Cassel (1991), Schoorl et al. (2002) e Zebarth et al. (2002), encontraram relação de atributos físicos com diferentes posições na paisagem.

Considerando que o perfil e as curvaturas do terreno condicionam variabilidade espacial diferenciada dos atributos físicos do solo, esse estudo foi realizado com o objetivo de estudar a variabilidade espacial da porosidade total, macroporosidade, microporosidade e densidade do solo em um Latossolo Vermelho eutroférrico, sob cultivo de cana-de-açúcar, em áreas com variações da pedoforma.

\section{MATERIAL E MÉTODOS}

A área de estudo localiza-se no nordeste do estado de São Paulo, no município de Guariba, SP, cujas coordenadas geográficas são (21 ${ }^{\circ} 19^{\prime} \mathrm{S}$; 48 ${ }^{\circ} 13^{\prime} \mathrm{W}$; 600 m). O clima da região, segundo a classificação de Köppen, é do tipo mesotérmico com inverno seco (Cwa), precipitação média de $1400 \mathrm{~mm}$ e com chuvas concentradas no período de novembro a fevereiro. A vegetação natural se constituía por floresta tropical subcaducifólia e mata ciliar.

O relevo é predominantemente suave ondulado com declividades médias variando de 3 a $8 \%$. A área experimental está sob cultivo de cana-de-açúcar há mais de trinta anos. O solo da área foi classificado como Latossolo Vermelho eutroférrico textura muito argilosa (LVef) (EMBRAPA, 1999).

De acordo com o modelo de Troeh (1965), classificou-se a curvatura e o perfil das formas do terreno no terço inferior da encosta, em dois compartimentos (I e II), como mostrado na Figura 1. No compartimento I a forma do relevo é basicamente linear e o compartimento II apresenta curvaturas côncavas e convexas. Os solos foram amostrados nos pontos de cruzamento de uma malha, com intervalos regulares de $10 \mathrm{~m}$, nas profundidades de 0 - 0,2; 0,2 - 0,4 e 0,4 - 0,6 m, com dimensão de $100 \times 100 \mathrm{~m}$, totalizando 100 pontos.

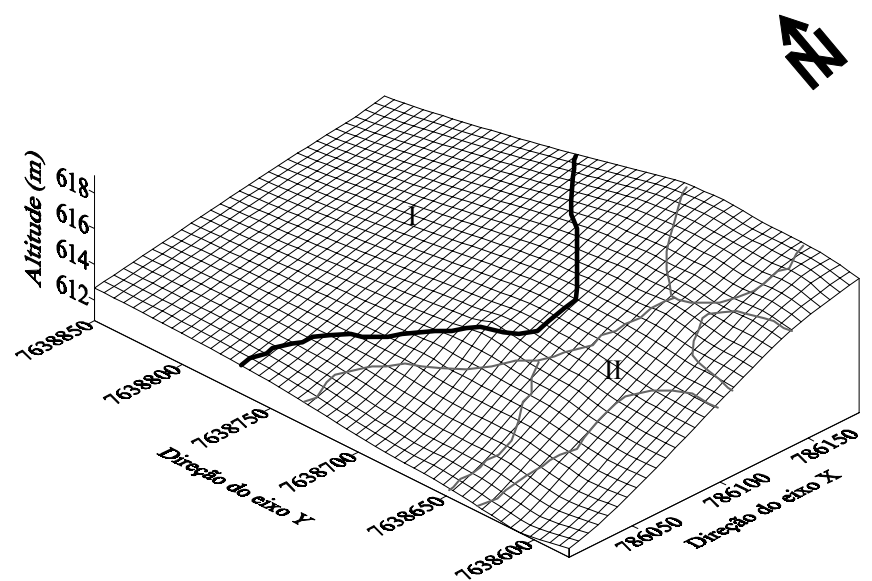

Figura 1. Modelo de elevação digital da área de estudo, com compartimentos identificados (I e II)

Foram abertas 100 trincheiras de $0,60 \mathrm{~m}$ de profundidade (0,3 x 0,3 m de largura), para coleta de amostras indeformadas em anéis volumétricos, para determinação dos atributos físicos. Para a determinação da porosidade do solo (porosidade total, macro e microporosidade), as amostras indeformadas foram saturadas por $48 \mathrm{~h}$ em bandeja com água até dois terços da altura do anel. Após o período de saturação, as amostras foram drenadas no potencial equivalente a-0,006 MPa utilizando-se uma mesa de tensão (EMBRAPA, 1997). Nas mesmas amostras determinou-se a densidade do solo, através do método do volume conhecido (EMBRAPA, 1997).

A variabilidade do solo foi, primeiramente, avaliada pela análise da média, mediana, variância, coeficiente de variação, coeficiente de assimetria e coeficiente de curtose, máximo e mínimo. A hipótese de normalidade dos dados foi testada pelo teste de Kolmogorov-Smirnov e a análise descritiva dos dados foi realizada usando-se o programa SAS (1995). A observação de valores extremos (máximos e mínimos) e a distribuição de freqüência revelaram a existência de valores atípicos para as variáveis em estudo. Após a verificação da inexistência de erros dos dados de laboratório, optou-se pela reedição dos dados extremos, através da média de seus vizinhos.

A análise da dependência espacial foi feita por meio da geoestatística, conforme Vieira et al. (1983) e Robertson (1998) e do ajuste de semivariogramas. A análise da dependência espacial é baseada na suposição de que medições separadas por distâncias pequenas são mais semelhantes umas às outras, que aquelas separadas por distâncias maiores. A semivariância é, por definição, dada por:

$$
\gamma(h)=\frac{1}{2} E\left[Z\left(x_{i}\right)-Z\left(x_{i}+h\right)\right]^{2}
$$


a qual é uma função do vetor h e, portanto, depende da magnitude e da direção h.

A semivariância é estimada através da expressão seguinte:

$$
\hat{\gamma}(h)=\frac{1}{2 N(h)} \sum_{i=1}^{N(h)}\left[Z\left(x_{i}\right)-Z\left(x_{i}+h\right)\right]^{2}
$$

sendo $\mathrm{N}(\mathrm{h})$ o número de pares de valores medidos $\mathrm{Z}\left(\mathrm{x}_{\mathrm{i}}\right), \mathrm{Z}\left(\mathrm{x}_{\mathrm{i}}+\right.$ h), separados por um vetor h. O gráfico de $\gamma(\mathrm{h})$ é denominado semivariograma. Do ajuste de um modelo matemático aos valores estimados de $\hat{\gamma}(\mathrm{h})$ são definidos os coeficientes do modelo teórico para o semivariograma (o efeito pepita, $\mathrm{C}_{0}$; patamar, $\mathrm{C}_{0}+\mathrm{C}_{1}$; e o alcance, a). $\mathrm{O}$ efeito pepita é o valor da semivariância para a distância zero e representa o componente da variação ao acaso; o patamar é o valor da semivariância onde a curva se estabiliza sobre um valor constante; o alcance é a distância da origem até onde o patamar atinge valores estáveis, considerando-se o limite da dependência espacial da grandeza medida. Foram ajustados aos dados os seguintes modelos: (a) esférico (Esf), $\hat{\gamma}(\mathrm{h})=\mathrm{C}_{0}+\mathrm{C}_{1}\left[1,5(\mathrm{~h} / \mathrm{a})-0,5(\mathrm{~h} / \mathrm{a})^{3}\right]$ para $0<\mathrm{h}<$ a e $\hat{\gamma}(\mathrm{h})=\mathrm{C}_{0}+\mathrm{C}_{1}$ para $\mathrm{h}>\mathrm{a}$; (b) exponencial (Exp), $\hat{\gamma}(\mathrm{h})=\mathrm{C}_{0}+\mathrm{C}_{1}[1-\exp (-3 \mathrm{~h} / \mathrm{a})]$ para $0<\mathrm{h}<\mathrm{d}$, em que d é a máxima distância na qual o semivariograma é definido; (c) gaussiano $(\mathrm{Gau}), \hat{\gamma}(\mathrm{h})=\mathrm{C}_{0}+\mathrm{C}_{1}\left[1-\exp \left(-3 \mathrm{~h}^{2} / \mathrm{a}^{2}\right)\right]$. A razão entre o efeito pepita e o patamar $\mathrm{C}_{0} /\left(\mathrm{C}_{0}+\mathrm{C}_{1}\right)$ expressa em porcentagem, permitiu a classificação do grau de dependência espacial, a qual segundo Cambardella et al. (1994) é considerada forte se esta razão for $\leq 25 \%$ do patamar, moderada quando ela está entre 25 e $75 \%$, e fraca se a razão for $>75 \%$.

Para se determinar a existência ou não da dependência espacial, ajustou-se semivariogramas, através do programa $\mathrm{GS}^{+}$ (Robertson, 1998). Em caso de dúvida entre mais de um modelo para o mesmo semivariograma, considerou-se o melhor $\mathrm{R}^{2}$ (coeficiente de determinação) e o menor SQR (soma de quadrados do resíduo). Para elaboração dos mapas de distribuição espacial das variáveis lançou-se mão do programa Surfer (1999).

\section{RESULTADOS E DISCUSSÃO}

Os resultados referentes à análise descritiva para as variáveis porosidade total, macroporosidade, microporosidade e densidade do solo, apresentados na Tabela 1, indicaram ajuste à distribuição normal. Os valores da média e mediana, para todas as variáveis, estão próximos, mostrando distribuições simétricas, o que pode ser confirmado pelos valores de assimetria próximos de zero.

O gráfico da probabilidade normal (Figura 2) permite verificar-se que as distribuições das variáveis porosidade total, macroporosidade, microporosidade e densidade do solo, apresentam comportamento próximo da linha reta; apenas a distribuição da variável porosidade total nas profundidades em estudo, afastou-se mais da reta nos extremos, evidenciandose pequeno afastamento da distribuição normal nas caudas. Os resultados referentes ao teste Kolmogorov-Smirnov
Tabela 1. Estatística descritiva para as variáveis porosidade total $\left(\mathrm{m}^{3} \mathrm{~m}^{-3}\right)$, macroporosidade $\left(\mathrm{m}^{3} \mathrm{~m}^{-3}\right)$, microporosidade $\left(\mathrm{m}^{3} \mathrm{~m}^{-3}\right)$ e densidade do solo $\left(\mathrm{kg} \mathrm{dm}^{-3}\right)$ de amostras coletadas na malha nas profundidades de $0-0,2 ; 0,2$ - 0,4 e 0,4-0,6 m

\begin{tabular}{|c|c|c|c|c|}
\hline \multirow{2}{*}{ Estatística } & \multicolumn{4}{|c|}{ Variáveis Físicas } \\
\hline & $\mathrm{PT}^{1}$ & Macro $^{2}$ & Micro $^{3}$ & $\mathrm{DS}^{4}$ \\
\hline \multicolumn{5}{|l|}{ A. $\quad 0-0,2 \mathrm{~m}$} \\
\hline Número & 100 & 100 & 100 & 100 \\
\hline Média & 0,51 & 0,14 & 0,37 & 1,40 \\
\hline Mediana & 0,52 & 0,14 & 0,38 & 1,41 \\
\hline Mínimo & 0,45 & 0,03 & 0,19 & 1,16 \\
\hline Máximo & 0,55 & 0,26 & 0,50 & 1,68 \\
\hline Variância & 0,008 & 0,003 & 0,005 & 0,014 \\
\hline Assimetria & $-0,39$ & 0,06 & $-0,48$ & 0,13 \\
\hline Curtose & $-0,79$ & $-0,80$ & $-0,40$ & $-0,53$ \\
\hline $\mathrm{CV}(\%)$ & 17,53 & 39,12 & 19,10 & 8,45 \\
\hline $\mathrm{p}^{5}$ & 0,10 & $0,05^{*}$ & 0,08 & $0,04^{*}$ \\
\hline \multicolumn{5}{|l|}{ B. $0,2-0,4 \mathrm{~m}$} \\
\hline Número & 100 & 100 & 100 & 100 \\
\hline Média & 0,51 & 0,12 & 0,38 & 1,41 \\
\hline Mediana & 0,52 & 0,12 & 0,39 & 1,40 \\
\hline Mínimo & 0,40 & 0,03 & 0,26 & 1,27 \\
\hline Máximo & 0,58 & 0,23 & 0,49 & 1,59 \\
\hline Variância & 0,002 & 0,001 & 0,002 & 0,006 \\
\hline Assimetria & $-0,60$ & 0,01 & $-0,45$ & 0,31 \\
\hline Curtose & $-0,79$ & $-0,31$ & $-0,08$ & $-0,60$ \\
\hline $\mathrm{CV}(\%)$ & 8,77 & 26,35 & 11,77 & 5,49 \\
\hline $\mathrm{p}$ & 0,14 & $0,04^{*}$ & $0,07^{*}$ & $0,07^{*}$ \\
\hline \multicolumn{5}{|l|}{ C. $0,4-0,6 \mathrm{~m}$} \\
\hline Número & 100 & 100 & 100 & 100 \\
\hline Média & 0,50 & 0,13 & 0,36 & 1,33 \\
\hline Mediana & 0,51 & 0,13 & 0,37 & 1,33 \\
\hline Mínimo & 0,41 & 0,09 & 0,22 & 1,17 \\
\hline Máximo & 0,59 & 0,21 & 0,48 & 1,49 \\
\hline Variância & 0,001 & 0,001 & 0,003 & 0,007 \\
\hline Assimetria & $-0,36$ & $-0,07$ & $-0,51$ & 0,35 \\
\hline Curtose & $-0,70$ & $-0,26$ & $-0,25$ & $-0,45$ \\
\hline $\mathrm{CV}(\%)$ & 6,32 & 24,32 & 16,21 & 6,29 \\
\hline $\mathrm{p}$ & 0,08 & $0,08^{*}$ & 0,09 & $0,07^{*}$ \\
\hline
\end{tabular}

${ }^{1} \mathrm{PT}=$ Porosidade total

${ }^{2}$ Macro $=$ Macroporosidade

${ }^{3}$ Micro $=$ Microporosidade

${ }^{4} \mathrm{DS}=$ Densidade do solo

${ }^{5} \mathrm{p}=$ Estatística do teste de Kolmogorov-Smirnov, significativo a $5 \%$ de probabilidade $(*)$

indicaram normalidade para as variáveis macroporosidade e densidade do solo, em todas as profundidades estudadas, e microporosidade, na profundidade de 0,2 - 0,4 m; a normalidade para a variável densidade do solo também foi verificada por Souza et al. (2001) e Carvalho et al. (2003) e macroporosidade por Mata et al. (1998).

Segundo Isaaks \& Srivastava (1989) mais importante que a normalidade dos dados é a ocorrência ou não do chamado efeito proporcional, em que a média e a variabilidade dos dados sejam constantes na área de estudo, o que foi observado, ou seja, ocorre a estacionaridade necessária ao uso da geoestatística. No entanto, em se tratando de dados obtidos na natureza, o ajuste de uma distribuição teórica é apenas aproximado (Warrick \& Nielsen, 1980 e Goovaerts, 1997). A normalidade dos dados não é uma exigência da geoestatística; é conveniente apenas que a distribuição não apresente caudas muito alongadas, o que poderia comprometer as análises (Webster, 1985; Cressie, 1991). Nota-se, na Figura 2, para as variáveis em 

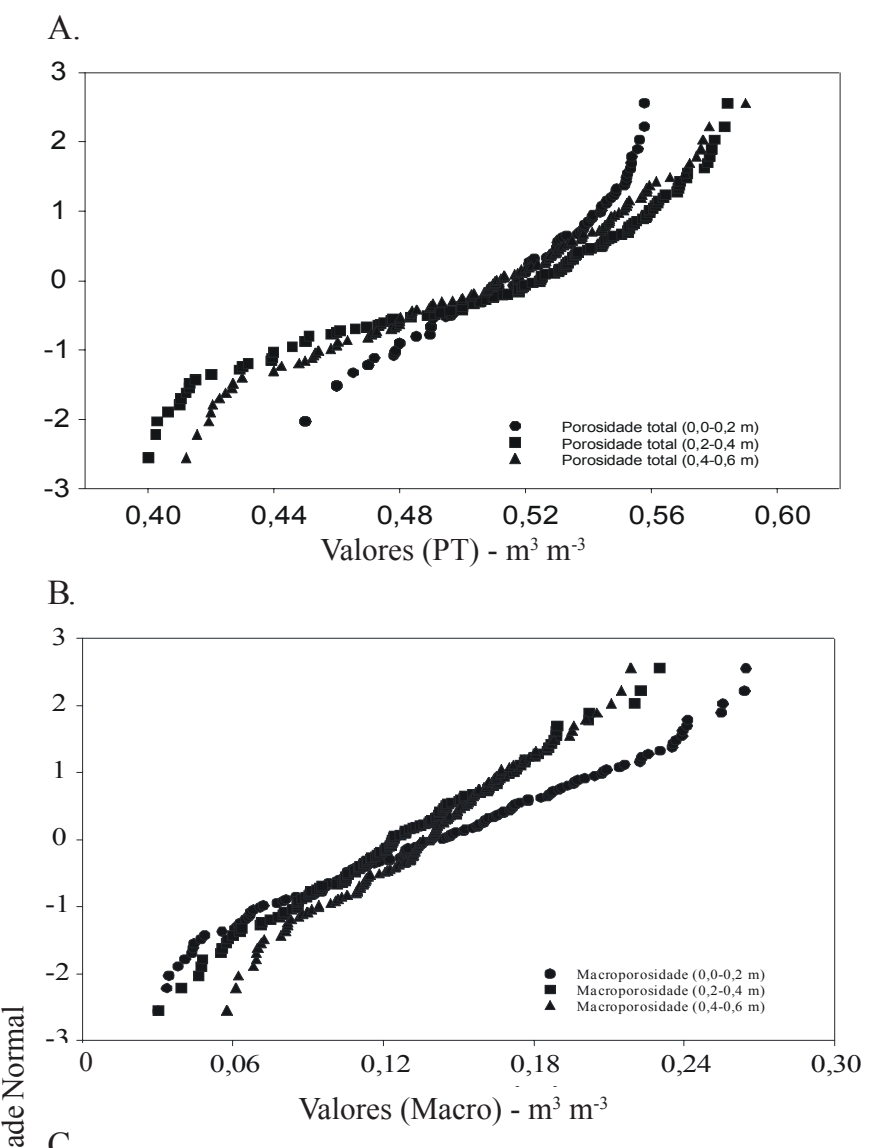

C.

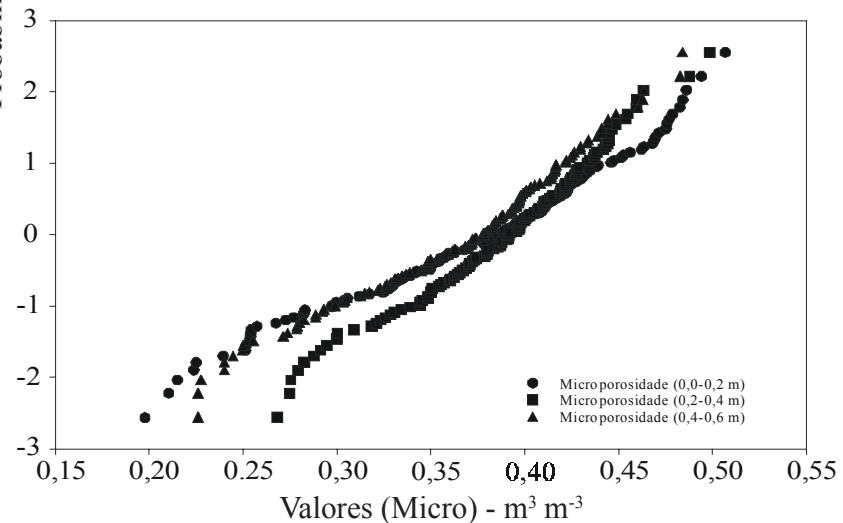

D.

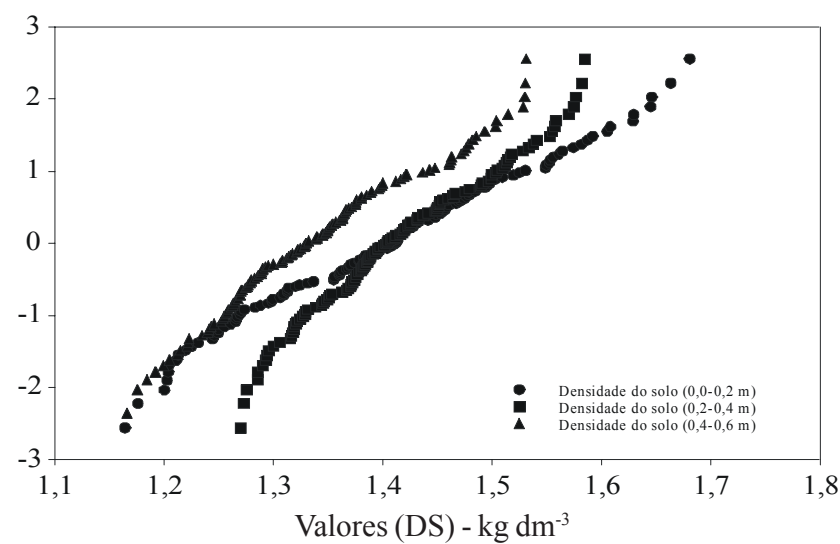

Figura 2. Gráficos de probabilidade normal para as variáveis

(A) PT - porosidade total, (B) macroporosidade - Macro,

(C) microporosidade - Micro e (D) densidade do solo - DS

nas profundidades de $0-0,2 ; 0,2-0,4$ e $0,4-0,6 \mathrm{~m}$ estudo, que as distribuições são suficientemente simétricas para aplicação da geoestatística.

A densidade do solo na profundidade de $0,2-0,4 \mathrm{~m}$ apresentou o menor coeficiente de variação $(5,49 \%)$ enquanto a macroporosidade na profundidade de $0,0-0,2 \mathrm{~m}$, indicou o maior coeficiente de variação $(39,12 \%)$ (Tabela 1$)$. Adotandose o critério de classificação para $\mathrm{CV}$ proposto por Warrick \& Nielsen (1980) para medida estatística de dispersão, seus valores se revelaram: (a) alto ( $>24 \%$ ) para a variável macroporosidade, nas profundidades 0 - 0,2;0,2 - 0,4 e 0,4-0,6 m, concordando com os resultados obtidos por Machado (1994), Mata et al. (1998), Cavalcante (1999) e Carvalho et al. (2003) nas profundidades de 0 - 0,2 e 0,2 - 0,4 m; (b) médio (12 a 24\%) para a variável microporosidade nas profundidades de 0 - 0,2 m e 0,4 - 0,6 m; resultados semelhantes também foram encontrados por Carvalho et al. (2003) e porosidade total na profundidade de 0 - $0,2 \mathrm{~m}$; (c) baixo (<12\%) para a variável porosidade total nas profundidades de $0,2-0,4$ e $0,4-0,6 \mathrm{~m}$, microporosidade na profundidade de $0,2-0,4 \mathrm{~m}$ e densidade do solo em todas as profundidades estudadas. Esses resultados estão de acordo com os obtidos por Machado (1994), Cavalcante (1999), Souza et al. (2001) e Carvalho et al. (2003). Outros autores têm adotado essas classes de $\mathrm{CV}$ em trabalhos relacionados à variabilidade espacial do solo (Libardi et al., 1986; Macedo et al., 1998; Salviano et al., 1998, Gonçalves et al., 2001). Apesar dos coeficientes de variação permitirem que se compare a variabilidade entre amostras com unidades diferentes, seu emprego não deve ser generalizado, devendo-se apreciar esses resultados segundo as finalidades a que se destina o trabalho (Oliveira \& Menk, 1974).

Os resultados da análise geoestatística (Tabela 2 e Figura 3) mostraram que todas as variáveis analisadas apresentaram dependência espacial nas profundidades em estudo, com exceção das variáveis macroporosidade e densidade do solo, na profundidade de $0,4-0,6 \mathrm{~m}$. Na profundidade $0,0-0,2 \mathrm{~m}$, todas as variáveis se ajustaram ao modelo esférico, concordando com os resultados obtidos por Cavalcante (1999) e Souza et al. (2001); na profundidade de 0,2-0,4 $\mathrm{m}$ as variáveis macroporosidade e microporosidade ajustaram-se ao modelo esférico, de acordo com os dados de Souza et al. (2001) para a microporosidade e Carvalho et al. (2003) para a macroporosidade e as variáveis porosidade total e densidade do solo ao modelo exponencial; na profundidade de $0,4-0,6 \mathrm{~m}$, as variáveis porosidade total e microporosidade ajustaram-se ao modelo esférico. Para as variáveis que apresentaram efeito pepita puro, ou seja, ausência de dependência espacial, distribuição espacial aleatória, a estatística clássica pode ser aplicada.

O efeito pepita como percentagem do patamar, permite que se compare o grau da dependência espacial de variáveis estudadas (Trangmar et al., 1985). Segundo a classificação de Cambardella et al. (1994) todas as variáveis estudadas apresentaram dependência espacial forte, com exceção da variável porosidade total na profundidade de 0,2 - 0,4 m. Cambardella et al. (1994), afirmam que as variáveis que apresentam forte dependência espacial são mais influenciadas por propriedades intrínsecas do solo, como os fatores de formação do solo (material de origem, relevo, clima, organismos e tempo). 
Tabela 2. Modelos e parâmetros estimados dos semivariogramas experimentais para as variáveis porosidade total $\left(\mathrm{m}^{3} \mathrm{~m}^{-3}\right)$, macroporosidade $\left(\mathrm{m}^{3} \mathrm{~m}^{-3}\right)$, microporosidade $\left(\mathrm{m}^{3} \mathrm{~m}^{-3}\right)$ e densidade do solo $\left(\mathrm{kg} \mathrm{dm}^{-3}\right)$ de amostras coletadas na malha, nas profundidades de 0 - 0,2;0,2 - 0,4 e 0,4 - 0,6 m

\begin{tabular}{|c|c|c|c|c|}
\hline \multirow{2}{*}{ Parâmetro* } & \multicolumn{4}{|c|}{ Variáveis Físicas } \\
\hline & PT & MACRO & MICRO & DS \\
\hline \multicolumn{5}{|l|}{ A. $0,0-0,2 \mathrm{~m}$} \\
\hline Modelo & Esférico & Esférico & Esférico & Esférico \\
\hline $\mathrm{C}_{0}$ & 0,0001 & 0,0006 & 0,0004 & 0,002 \\
\hline $\mathrm{C}_{0}+\mathrm{C}_{1}$ & 0,0008 & 0,003 & 0,002 & 0,008 \\
\hline $\mathrm{a}$ & 27 & 20 & 27 & 20 \\
\hline$\left[\mathrm{C}_{0} /\left(\mathrm{C}_{0}+\mathrm{C}_{1}\right)\right] \mathrm{x} 100$ & 12,5 & 20 & 20 & 25 \\
\hline $\mathrm{R}^{2}(\%)$ & 97 & 92 & 90 & 90 \\
\hline SQR & $1,10 \mathrm{E}-08$ & 3,66 E-08 & 1,97 E-07 & 8,34 E-07 \\
\hline \multicolumn{5}{|l|}{ B. $0,2-0,4 \mathrm{~m}$} \\
\hline Modelo & Exponencial & Esférico & Esférico & Exponencial \\
\hline $\mathrm{C}_{0}$ & 0,0004 & 0,0001 & 0,0003 & 0,0001 \\
\hline $\mathrm{C}_{0}+\mathrm{C}_{1}$ & 0,001 & 0,0009 & 0,002 & 0,002 \\
\hline $\mathrm{a}$ & 47 & 21 & 34 & 70 \\
\hline$\left[\mathrm{C}_{0} /\left(\mathrm{C}_{0}+\mathrm{C}_{1}\right)\right] \times 100$ & 40 & 11 & 15 & 5 \\
\hline $\mathrm{R}^{2}(\%)$ & 90 & 87 & 91 & 94 \\
\hline SQR & 3,45 E-08 & $1,41 \mathrm{E}-08$ & 6,63 E-08 & 5,71 E-08 \\
\hline \multicolumn{5}{|l|}{ C. $0,4-0,6 \mathrm{~m}$} \\
\hline Modelo & Esférico & $\begin{array}{l}\text { Pepita } \\
\text { puro }\end{array}$ & Esférico & Pepita puro \\
\hline $\mathrm{C}_{0}$ & 0,0005 & - & 0,0006 & - \\
\hline $\mathrm{C}_{0}+\mathrm{C}_{1}$ & 0,002 & - & 0,0026 & - \\
\hline $\mathrm{a}$ & 80 & - & 30 & - \\
\hline$\left[\mathrm{C}_{0} /\left(\mathrm{C}_{0}+\mathrm{C}_{1}\right)\right] \times 100$ & 25 & - & 23 & - \\
\hline $\mathrm{R}^{2}(\%)$ & 97 & - & 92 & - \\
\hline SQR & 2,4 E-08 & - & 7,31 E-08 & - \\
\hline
\end{tabular}

${ }^{*} \mathrm{PT}=$ Porosidade total MACRO $=$ Macroporosidade MICRO $=$ Microporosidade; $\mathrm{DS}=$ Densidade do solo; $\mathrm{C}_{0}=$ Efeito pepita; $\mathrm{C}_{0}+\mathrm{C}_{1}=$ Patamar; $\mathrm{a}=$ Alcance; $\mathrm{C}_{0} /\left(\mathrm{C}_{0}+\mathrm{C}_{1}\right)=$ Grau de dependência espacial, $\mathrm{R}^{2}=$ Coeficiente de determinação e $\mathrm{SQR}=$ Soma dos quadrados dos resíduos

O alcance estabelece o limite de dependência espacial entre as amostras, isto é, para distâncias iguais ou menores que o alcance, diz-se que os valores vizinhos de uma variável estão espacialmente correlacionados e podem ser utilizados para se estimar valores em qualquer ponto entre eles (Reichardt, 1985; Trangmar et al., 1985). As variáveis apresentaram diferentes alcances entre as profundidades de 0 - 0,2; 0,2 - 0,4 e 0,4 - 0,6 m; a variável prosidade total mostrou o maior alcance $(80 \mathrm{~m})$ na profundidade de 0,4 - 0,6 $\mathrm{m}$ e a densidade do solo e macroporosidade, os menores alcances $(20 \mathrm{~m})$ na profundidade de 0 - 0,20 $\mathrm{m}$. Na profundidade de $0-0,2 \mathrm{~m}$, os alcances foram os menores quando comparados com a profundidade de 0,2 - 0,4 m, ocorrendo aumento do alcance em profundidade para todas as variáveis em estudo, indicando a maior descontinuidade na distribuição espacial das propriedades do solo na camada mais superficial, concordando com estudo de Souza et al. (2001).

Os parâmetros dos modelos de semivariogramas ajustados foram utilizados para se estimar valores em locais não amostrados através da krigagem (Figura 4). Os valores da densidade do solo foram considerados altos, segundo Arshad et al. (1996), os quais relatam que valores acima $1,35 \mathrm{~kg} \mathrm{dm}^{-3}$ restringem o crescimento radicular em solo argiloso. A macroporosidade apresentou valores baixos, segundo Arshad et al. (1996), valores abaixo de $0,15 \mathrm{~m}^{-3} \mathrm{~m}^{-3}$ restringem o movimento de água no perfil do solo. Kiehl (1979) afirma que solos agrícolas nunca deveriam ter menos de $0,10 \mathrm{~m}^{-3} \mathrm{~m}^{-3} \mathrm{de}$ macroporos, sob pena de sério comprometimento da produtividade das culturas. Os valores altos para a densidade do solo e microporosidade e baixos para a macroporosidade $\mathrm{e}$ porosidade total, estão relacionados com o tráfego intensivo de máquinas na cultura da cana-de-açúcar, segundo Salire et al. (1994) e Hakansson \& Voorhees (1997) sistemas com pouco revolvimento do solo e tráfego de máquinas pesadas, pode promover compactação do solo até $0,4 \mathrm{~m}$.

Cruzando as informações da Figura 1 e os mapas de krigagem (Figura 4), observa-se que no compartimento I a área apresenta um relevo com forma linear e declividade homogênea apresentando variabilidade menor para as variáveis em estudo, já no compartimento II o relevo possui formas côncavas e convexas condicionando maior variabilidade das variáveis em estudo. Machado (1994) e Marques Júnior \& Lepsch (2000), afirmam que a posição topográfica dos solos na paisagem, influindo na drenagem do solo, é um dos principais fatores condicionantes da variabilidade espacial dos solos. Li et al. (2001) estudando a produção de algodão com auxílio da geoestatísitica e paisagem, verificaram que os atributos químicos e físicos têm forte relação com a paisagem. Pennock et al. (1992) e Boehm \& Anderson (1997) encontraram relação da densidade do solo com a paisagem e Sobieraj et al. (2002) com a macroporosidade do solo.

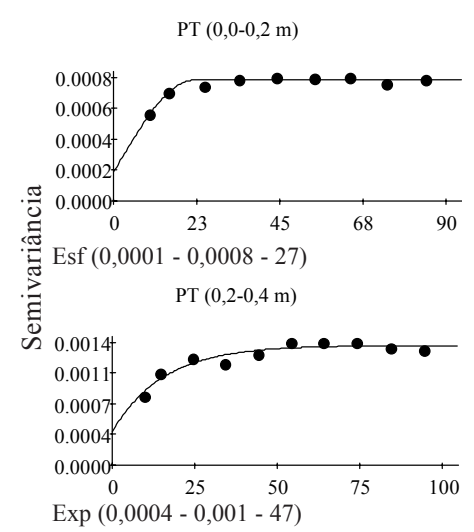

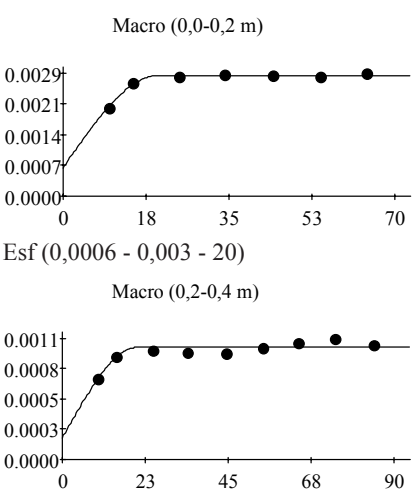

Esf $(0,0001-0,0009-21)$

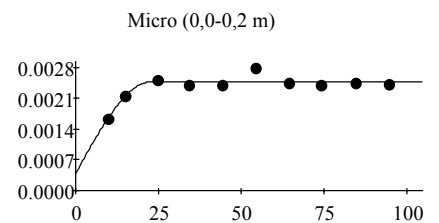

Esf $(0,0004-0,002-27)$

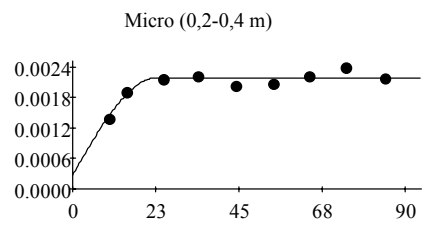

Esf $(0,0003-0,002-34)$

Distância $(\mathrm{m})$

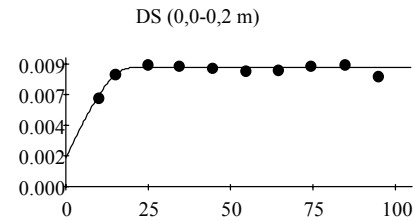

Esf $(0,002-0,008-20)$

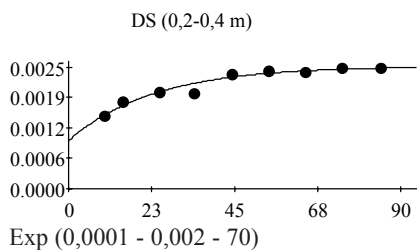

Legenda: Esf e Exp $\left(\mathrm{C}_{0} \mathrm{C}_{0}+\mathrm{C}_{1}\right.$; a) é o modelo esférico e exponencial ajustado, $\mathrm{C}_{0}=$ efeito pepita; $\mathrm{C}_{0}+\mathrm{C}_{1}=$ patamar; a $=$ alcance

Figura 3. Semivariogramas das variáveis físicas, PT - porosidade total $\left(\mathrm{m}^{3} \mathrm{~m}^{-3}\right)$, Macro - macroporosidade $\left(\mathrm{m}^{3} \mathrm{~m}^{-3}\right)$, Micro - microporosidade $\left(\mathrm{m}^{3} \mathrm{~m}^{-3}\right)$ e DS - densidade do solo $\left(\mathrm{kg} \mathrm{dm}^{-3}\right)$ nas profundidades de $0,0-0,2 \mathrm{~m}$ e $0,2-0,4 \mathrm{~m}$ 

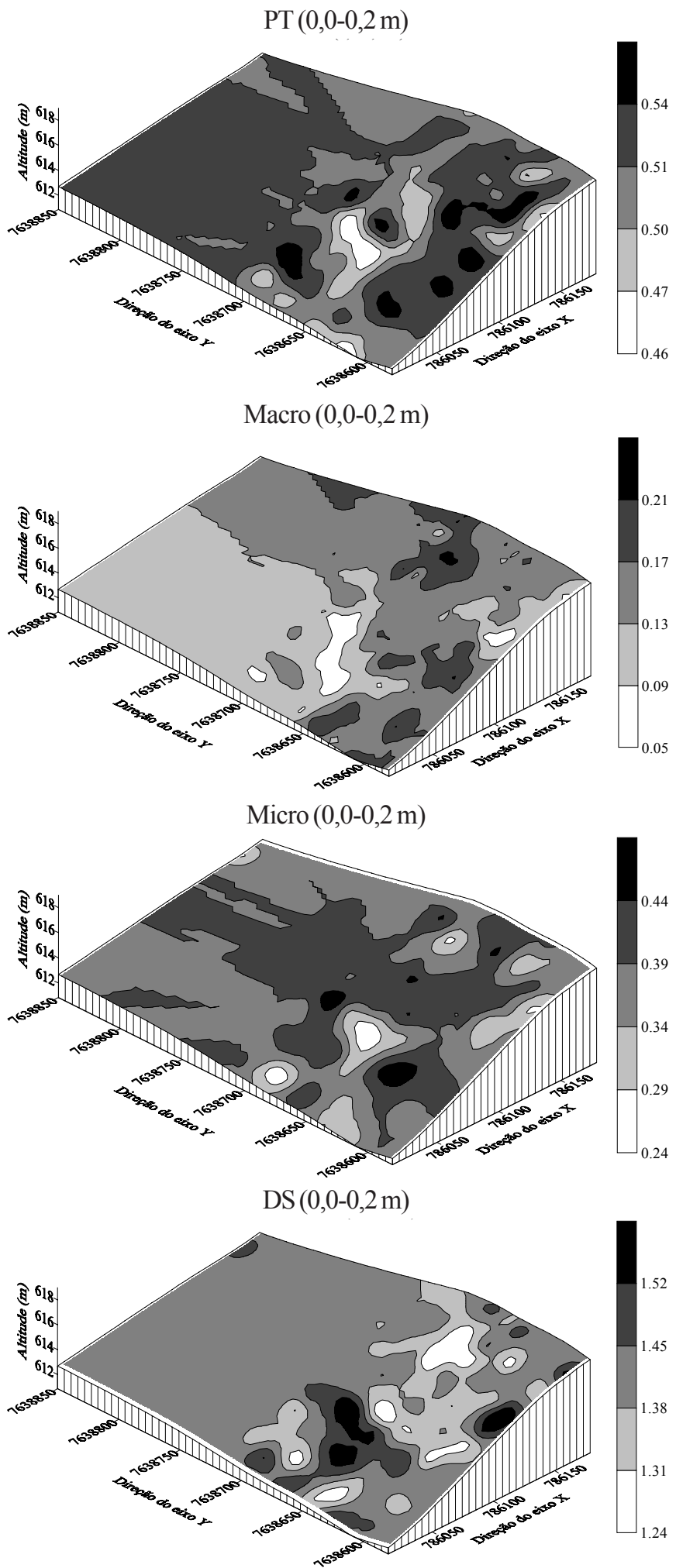

Figura 4. Distribuição espacial da porosidade total $\left(\mathrm{m}^{3} \mathrm{~m}^{-3}\right)$, macroporosidade $\left(\mathrm{m}^{3} \mathrm{~m}^{-3}\right)$, microporosidade $\left(\mathrm{m}^{3} \mathrm{~m}^{-3}\right)$ e densidade do solo $\left(\mathrm{kg} \mathrm{dm}^{-3}\right)$ de amostras coletadas na malha nas profundidades de 0 - 0,2 e 0,2 - 0,4 m

\section{CONCLUSÕES}

1. O coeficiente de variação indicou variabilidade baixa para as variáveis densidade do solo, em todas as profundidades estudadas, e para a porosidade total nas profundidades de 0,2
PT $(0,2-0,4 \mathrm{~m})$

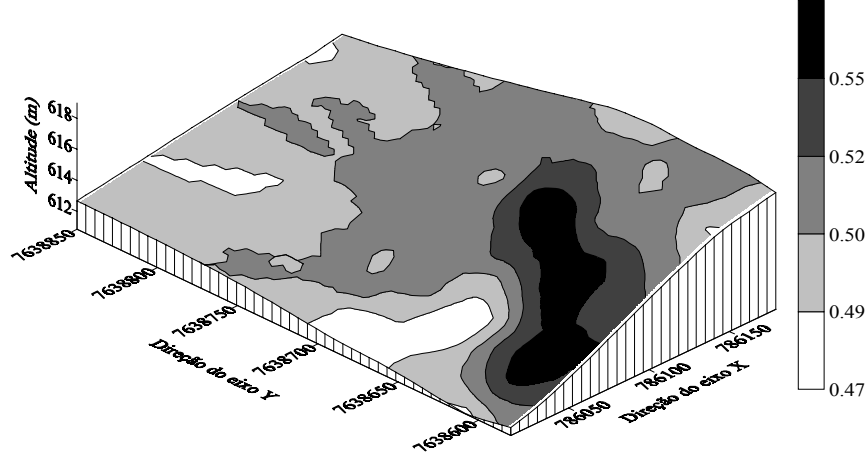

Macro $(0,2-0,4 \mathrm{~m})$

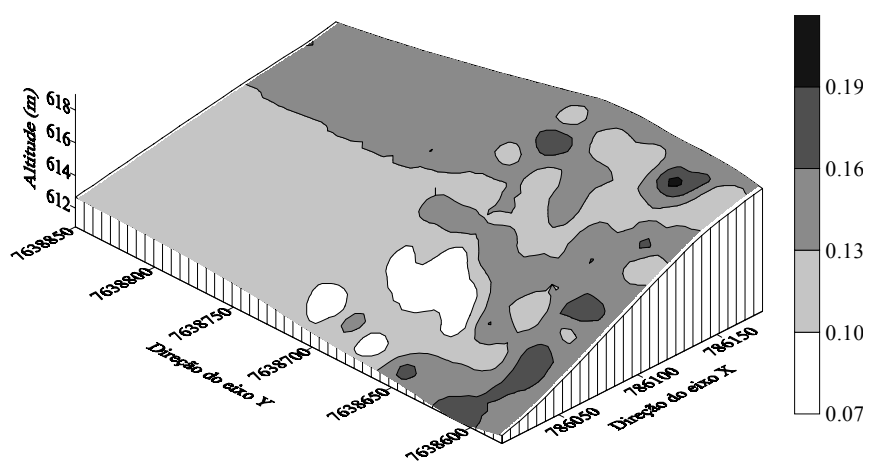

$\operatorname{Micro}(0,2-0,4 \mathrm{~m})$

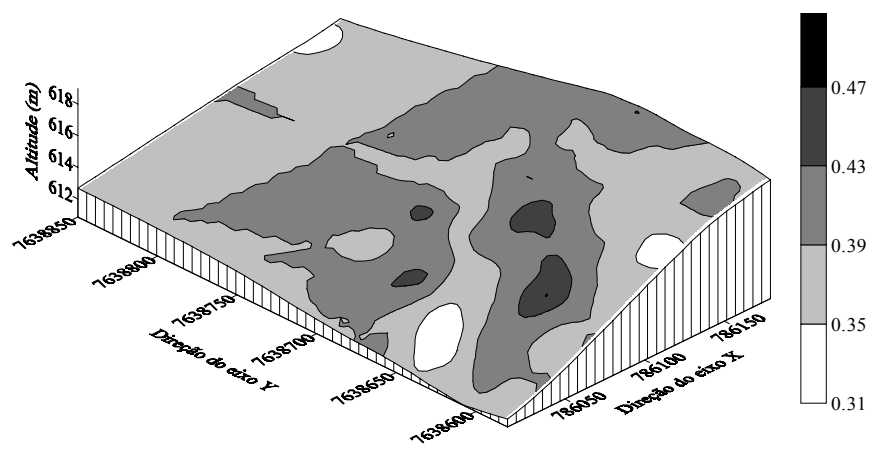

$\operatorname{DS}(0,2-0,4 \mathrm{~m})$

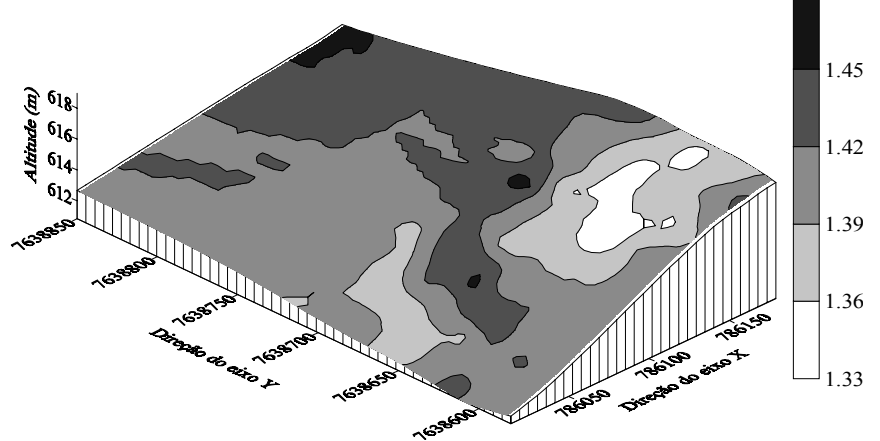


da variável porosidade total, na profundidade de $0,2-0,4 \mathrm{~m}$.

3 . Os maiores alcances foram observados nas profundidades de 0,2 - 0,4 e 0,4 - 0,6 m, para todos as variáveis estudadas.

4. A redução da porosidade total, microporosidade e, sobretudo, da macroporosidade, promoveu os altos valores para a densidade do solo, principalmente na profundidade de 0,2 - 0,4 m, indicando compactação nesta camada.

5. A identificação de compartimentos da paisagem na área de estudo mostrou-se muito eficiente na compreensão das causas da variabilidade espacial dos atributos estudados. Pequenas variações nas formas do relevo condicionam variabilidade diferenciada para os atributos físicos do solo.

\section{AGRADECIMENTOS}

À Fundação de Amparea à Pesquisa do Estado de São Paulo pela concessão de bolsa ao primeiro autor e auxílio financeiro para o desenvolvimento do projeto. À a Usina São Martinho pela concessão da área para o experimento.

\section{LITERATURA CITADA}

Albuquerque, J.A.; Reinert, D.J.; Florin, J.E.; Ruedell, J.; Petrere, C.; Fontinelli, F. Rotação de culturas e sistemas de manejo do solo: efeito sobre a forma da estrutura do solo ao final de sete anos. Revista Brasileira de Ciência do Solo, Campinas, v.19, n.1, p.115-119, 1995.

Arshad, M.A.; Lower, B.; Grossman, B. Physical tests for monitoring soil quality. In: Doran, J.W.; Jones, A.J. (eds.). Methods for assessing soil quality. Madison, Soil Science Society of America. 1996. p.123-141. SSSA Special publication 49

Bathke, G.R.; Cassel, D.K. Anisotropic variation of profile characteristics and saturated hydraulic conductivity in an ultisol landscape. Soil Science Society America Journal, Madison, v.55, n.2, p.333-339, 1991.

Boehm, M.M.; Anderson, D.W. A landscape-scale study of soil quality in three prairie farming systems. Soil Science Society America Journal, Madison, v.61, n.4, p.1147-1159, 1997.

Cambardella, C.A.; Moorman, T.B.; Novak, J.M.; Parkin, T.B.; Karlen, D.L.; Turco, R.F.; Konopka, A.E. Field-scale variability of soil properties in Central Iowa Soils. Soil Science Society America Journal, Madison, v.58, n.5, p.1501-1511, 1994.

Carvalho, J.R.P.; Silveira, P.M.; Vieira, S.R. Geoestatística na determinação da variabilidade espacial de características químicas do solo sob diferentes preparos. Pesquisa Agropecuária Brasileira, Brasília, v.37, n.8, p.1151-1159, 2002.

Carvalho, M.P.; Takeda, E.Y.; Freddi, O.S. Variabilidade espacial de atributos de um solo sob videira em Vitória Brasil (SP). Revista Brasileira de Ciência do Solo, Viçosa, v.27, n.4, p.695703, 2003.

Cavalcante, E.G.S. Variabilidade espacial de atributos físicos e químicos de um Latossolo Vermelho-Escuro sob diferentes condições de uso e manejo. Ilha Solteira: UNESP, 1999. 199p. Dissertação Mestrado
Cavenage, A.; Moraes, M.L.T; Alves, M.C.; Carvalho, M.A.C.; Freitas, M.L.M.; Buzetti, S. Alterações nas propriedades físicas de um Latossolo Vermelho-Escuro sob diferentes culturas. Revista Brasileira de Ciência do Solo, Viçosa, v.23, n.4, p.997-1003, 1999.

Cressie, N. Statistics for spatial data. New York: John Wiley, 1991.

EMBRAPA - Empresa Brasileira de Pesquisa Agropecuária. Manual de métodos de análises de solo. Rio de Janeiro: Ministério da Agricultura e do Abastecimento, 1997.212p.

EMBRAPA - Empresa Brasileira de Pesquisa Agropecuária. Centro Nacional de Pesquisa de Solos. Sistema Brasileiro de Classificação de Solos. Rio de Janeiro, 1999. 412p.

Gonçalves, A.C.A.; Folegatti, M.V.; Mata, J.D.V. Análises exploratória e geoestatística da variabilidade de propriedades físicas de um argissolo vermelho. Acta Scientiarum, Maringá, v.23, n.5, p.1149-1157, 2001.

Goovaerts, P. Geoestatistics for natural resources evaluation. 1 ed. New York: Oxford University Press, 1997. 483p.

Hakansson, I.; Voorhees, W.B. Soil compaction. In: Lal, R.; Blum, W.H.; Valentin, C. (ed.). Methods for assessment of soil degradation. Boca Raton: Lewis, 1997. p.167-179.

Isaaks, E.H.; Srivastava, R.M. An introduction to applied geoestatistics. New York: Oxford University Press, 1989. $561 p$.

Kiehl, E.J. Manual de Edafologia: relações solo-planta. 1.ed. São Paulo: Agronômica Ceres, 1979. 264p.

Klein, V.A.; Boller, W. Avaliação de diferentes métodos de manejos do solo e métodos de semeadura em área sob sistema de plantio direto. Ciência Rural, Santa Maria, v.25, n.3, p.395398, 1995.

Li, H.; Lascano, R.J.; Booker, J.; Wilson, L.T.; Bronson, K.F. Cotton lint yield variability in a heterogeneous soil at a landscape scale. Soil Tillage Research, Oxford, v.58, n.3-4, p.245-258, 2001.

Libardi, P.L.; Prevedello, C.L.; Pauletto, E.A.; Moraes, S.O. Variabilidade espacial da umidade, textura e densidade de partículas ao longo de uma transeção. Revista Brasileira de Ciência do Solo, Campinas, v.10, n.2, p.85-90, 1986.

Macedo, J.R.; Otonni Filho, T.B.; Meneguelli, N.A. Variabilidade de características físicas, químicas e físico-hídricas em solo Podzólico Vermelho-Amarelo de Seropédica, RJ. Pesquisa Agropecuária Brasileira, Brasília, v.33, n.12, p.2043-2053, 1998.

Machado, R.V. Variabilidade espacial de atributos físicohídricos em uma hidrosseqüência de solos bem a muito mal drenados. Lavras: Escola Superior de Agricultura de Lavras, 1994. 88p. Dissertação Mestrado

Marques Júnior, J.; Lepsch, I.F. Depósitos superficiais neocenozóicos, superfícies geomórficas e solos em Monte Alto, SP. Geociências, São Paulo, v.19, n.2, p.265-281, 2000.

Mata, J.D.V.; Gonçalves, A.C.A.; Vieira, S.R. Variabilidade espacial da macroporosidade do solo em área irrigada, antes do preparo e após colheita, sob dois sistemas de preparo. Acta Scientiarum, Maringá, v.20, n.3, p.307-312, 1998.

Oliveira, J.B.; Menk, J.R.F. Variabilidade das características químicas e físicas em duas áreas homogêneas de Oxissolo. In: Congresso Brasileiro de Ciência do Solo, 14, Santa Maria, 1974. Anais... Campinas, Sociedade Brasileira de Ciência do Solo, 1974. p.359-376. 
Pennock, D.J.; Vankessel, C.; Farrell, R.E.; Sutherland, R.A. Landscape-scale variations in denitrification. Soil Science Society America Journal, Madison, v.56, n.3, p.770-776, 1992.

Reichardt, K. Processos de transferência no sistema soloplanta-atmosfera. Campinas, Fundação Cargill, 1985. 445p.

Robertson, G.P. GS ${ }^{+}$: Geostatistics for the environmental sciences $-\mathrm{GS}^{+}$User's Guide. Plainwell, Gamma Design Soffware, 1998. 152p.

Salire, E.V.; Hammen, J.E.; Hardcastle, J.H. Compression of intact subsoils under short-duration loading. Soil Tillage Research, Oxford, v.31, n.2-3, p.235-248, 1994.

Salviano, A.A.C.; Vieira, S.R.; Sparovek, G. Variabilidade espacial de atributos de solo e de Crotalaria juncea $(L)$ em área severamente erodida. Revista Brasileira de Ciência do Solo, Viçosa, v.22, n.1, p.115-122, 1998.

SAS, Statistical Analysis System for Windows. Computer program manual. Cary, 1995.

Schaefer, C.E.R.; Souza, C.M.; Vallejos, M.; Viana, J.H.M., Galvão, J.C.C.; Ribeiro, L.M. Características da porosidade de um Argissolo Vermelho-Amarelo submetido a diferentes sistemas de preparo de solo. Revista Brasileira de Ciência do Solo, Viçosa, v.25, n.3, p.765-769, 2001.

Schoorl, J.M.; Veldkamp, A.; Bouma, J. Modeling water and soil redistribution in a dynamic landscape context. Soil Science Society America Journal, Madison, v.66, n.5, p.1610$1619,2002$.

Sobieraj, J.A.; Elsenbeer, H.; Coelho, R.M.; Newton, B. Spatial variability of soil hydraulic conductivity along a tropical rainforest catena. Geoderma, Amsterdam, v.108, n.1-2, p.7990, 2002.

Souza, Z.M.; Silva, M.L.S.; Guimarães, G.L.; Campos, D.T.S.; Carvalho, M.P.; Pereira, G.T. Variabilidade espacial de atributos físicos em um Latossolo Vermelho Distrófico sob semeadura direta em Selvíria, MS. Revista Brasileira de Ciência do Solo, Viçosa, v.25, n.3, p.699-707, 2001.
Stone, L.F.; Silveira, P.M. Efeitos do sistema de preparo e da rotação de culturas na porosidade e densidade do solo. Revista Brasileira de Ciência do Solo, Viçosa, v.25, n.2, p.395401, 2001.

Surfer. Surfer 7.0. Contouring and 3D surface mapping for scientist's engineers. User's Guide. New York: Golden software, Inc, 1999, 619p.

Trangmar, B.B.; Yost, R.S.; Wade, M.K.; Uehara, G. Applications of geostatistics to spatial studies of soil properties. Advances in Agronomy, San Diego, v.38, n.1, p.45-94, 1985.

Troeh, F.R. Landform equations fitted to contour maps. American Journal Science, New Haven, v.263, n.3, p.616$627,1965$.

Utset, A.; Cid, G. Soil penetrometer resistance spatial variability in a ferralsol at several soil moisture conditions. Soil Tillage Research, Oxford, v.61, n.1, p.193-202, 2001.

Vaidya, P.H.; Pal, D.K. Microtopography as a factor in the degradation of Vertisols in central India. Land Degradation Development, Amsterdam, v.13, n.5, p.429-445, 2002.

Vieira, S.R.; Hatfield, J.L.; Nielsen, D.R.; Biggar, J.W. Geoestatiscal theory and application to variability of some agronomical properties. Hilgardia, Oakland, v.51, n.1, p.1$75,1983$.

Zebarth, B.J.; Rees, H.; Walsh, J.; Chow, L.; Pennock, D.J. Soil variation within a hummocky podzolic landscape under intensive potato production. Geoderma, Amsterdam, v.110, n.1-2, p.19-33, 2002.

Warrick, A.W.; Nielsen, D.R. Spatial variability of soil physical properties in the field. In:Hillel, D. (ed.). Applications of soil physics. New York: Academic Press, 1980.

Webster, R. Quantitative spatial analysis of soil in the field. Advances in Soil Science, New York, v.3, n.1, p.1-70, 1985. 